\title{
Latent Topics-based Relevance Feedback for Video Retrieval
}

\author{
Ruben Fernandez-Beltran, Filiberto Pla \\ Institut of New Imaging Technologies, Universitat Jaume I, SPAIN
}

\begin{abstract}
This work presents a novel Content-Based Video Retrieval approach in order to cope with the semantic gap challenge by means of latent topics. Firstly, a supervised topic model is proposed to transform the classical retrieval approach into a class discovery problem. Subsequently, a new probabilistic ranking function is deduced from that model to tackle the semantic gap between low-level features and high-level concepts. Finally, a shortterm relevance feedback scheme is defined where queries can be initialised with samples from inside or outside the database. Several retrieval simulations have been carried out using three databases and seven different ranking functions to test the performance of the presented approach. Experiments revealed that the proposed ranking function is able to provide a competitive advantage within the content-based retrieval field.
\end{abstract}

Keywords:

Content-Based Video Retrieval, Relevance Feedback, Latent Topics, probabilistic Latent Semantic Analysis (pLSA), Information Retrieval 


\section{Introduction}

The low cost of image/video capture technology together with the increasing capacity of storage is producing a huge expansion of video collections. In this scenario, one of the most important challenges is how to retrieve users' relevant data from this vast amount of information. Content-Based Video Retrieval (CBVR) is concerned about providing users with those videos which satisfy their queries by means of the video content analysis. As a result, the CBVR field has become a very important research area and a wide variety of CBVR systems have been developed $[1,2,3,4]$. The standard CBVR procedure involves three main components: (i) a query, containing a few video examples of the semantic concept that the user is looking for; (ii) a database, which is used to retrieve videos related to the query concept; and (iii) a ranking function, which sorts the database according to the relevance with respect to the user's query. These three components are typically integrated with the user in a Relevance Feedback (RF) scheme [5] to provide the most relevant videos through several feedback iterations.

Figure 1 shows the general RF scheme for retrieval. At the initialisation stage (stage 0), the user introduces the query concept into the system by providing $Q$ examples of the concept of interest. Then, the interactive process consists of the alternation of two stages through $I$ feedback iterations. In the retrieval stage (stage 1), the system ranks the database according to the query and shows the $S$ top items (scope) to the user. In the feedback stage (stage 2), the user checks the scope to select the correctly retrieved samples and finally the query is expanded with these new positive examples to carry out the next iteration. The ranking function can be considered the 


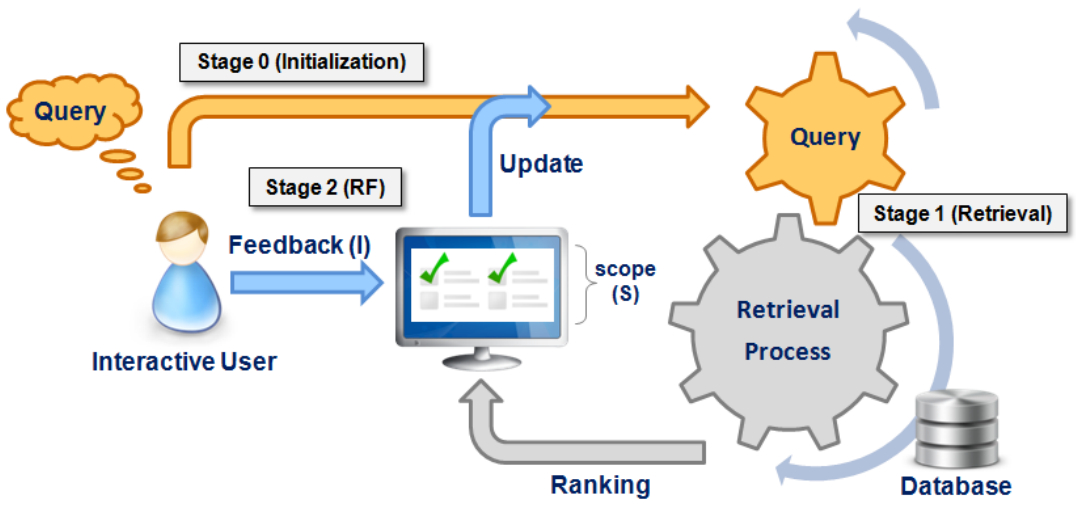

Figure 1: Relevance Feedback scheme. $Q$ is the number of initial examples in the query, $I$ the number of feedback iterations and $S$ the number of top ranked samples.

kernel of the retrieval system because it is in charge of scoring the samples of the database according to the query. As a result, the nature of the ranking function and the nature of the video representation space where the ranking function works are two of the most important factors for the precision of a CBVR system.

\subsection{Ranking functions}

One of the most common rankings in multimedia retrieval is the distancebased ranking. Such ranking is performed according to the minimum distance or maximum similarity in the video representation space. Several functions have been proposed in the content-based retrieval field. For instance, in [6] an image retrieval systems is presented which is based on an Euclidean ranking of micro-structure features that combine color, texture and shape. In other works, such as [7], the retrieval ranking is performed using combinations of similarity measures. Even, some authors [8] have combined several descriptors and distance measures to rank the database. Nevertheless, these kinds 
of functions tend to perform poorer when the query concepts to retrieve are rather complex [9].

Other ranking algorithms are based on inductive learning $[10,11]$ which typically use a bank of classifiers to represent the set of possible events to test. However, this approach usually leads to a constrained retrieval scheme where users are not allowed to search whatever they want. The CBVR problem itself has an unconstrained nature $[9,12]$ because the concept to retrieve is a priori unknown. Moreover, the performance of these methods highly depends on the used training data but in the CBVR application the initialisation and feedback are often too limited to provide a consistent training set.

Alternative ranking methods are based on transductive ranking. They use the own topology of the data to improve the output ranking. One of the most representative ones is Manifold Ranking (MR) [13] which ranks the data with respect to the intrinsic data distribution. In a more recent work [14], Yang et al. present a new transductive ranking function called Local Regression and Global Alignment (LRGA) to learn a robust Laplacian matrix which is able to slightly improve the performance of MR. The main drawback of these methods is their high computational cost because they require demanding matrix operations over the retrieval process. Transductive ranking functions are usually applied in the original descriptor space, however other authors have used a different representation space to perform the ranking. In [15], Zhang et al. present an image retrieval system which computes the cosine similarity function in a topic space to rank the database. This work uses positive (checked) and negative (unchecked) samples in the interactive retrieval process, but managing negative samples adds an extra 
effort because users have to check false negatives in addition to true positives.

\subsection{Video representation space}

Ranking functions run in a specific representation space where videos are encoded in feature vectors according to the information provided by a descriptor. In the literature, different kinds of descriptors have been proposed using static information - Scale Invariant Feature Transform (SIFT) [16]), spatio-temporal - Spatial Temporal Interest Points (STIP) [17]) or audio Mel Frequency Cepstral Coefficients (MFCC) [18]. The standard procedure to encode all this low-level information in feature vectors is the visual Bag of Words (vBoW) [19]. The vBoW quantisation starts by learning a visual vocabulary made up of the clustering of the local features. Then, each video is represented in a single histogram of visual words by accumulating the number of local features into their closest clusters. Authors usually refer to this quantised space as descriptor space although it is not the direct output of the descriptor functions. Some recent works have presented more advanced descriptors which are able to achieve better results for specific applications. Wang and Schmid [20] presented a video representation based on dense tra-

jectories specially designed for action recognition which outperforms the most common motion-based descriptors. However, in unconstrained CBVR the type of concepts to deal with is so wide that simpler and non-specialised descriptors are commonly used [4].

\subsection{Limitations of current approaches and topic models}

Several of the aforementioned approaches have shown to be successful at retrieval tasks when they are used on reduced databases with a small 
number of concepts [21]. Nonetheless, the so-called semantic gap [22] between computable low-level features and query concepts is still a challenge for huge unconstrained video collections. The visual variability of semantic concepts is so high that often current approaches are not able to capture properly unconstrained queries in extensive collections [4]. Therefore, new capabilities are required in CBVR to bring the video characterisation to a higher semantic level.

Although early research on topic models suggested that they may be used in video retrieval, it was not until recently that topic models were successfully applied to large unconstrained video collections [23]. In general, topic models can be used for automatically organising, understanding, searching and summarising large electronic archives [24]. For many years, topic models have not been considered useful in tasks where precision is important because traditional ranking functions tend to perform worse in the latent space than in the original characterisation space [25]. The latent topic space is usually a lower dimensionality representation where concepts and classes are more diffuse and besides it allows connections among different concepts through the patterns defined by topics. As a result, the most effective ranking functions in the original feature space are usually not useful in the topic space because this space has an utterly different nature. However, this fact does not mean the topics' lack of usefulness. In those applications in which the semantic gap is important, the retrieval precision in the original feature space tends to be very low and topic models can provide a competitive advantage by means of the hidden patterns that topics represent. It is the case of unconstrained CBVR. The difference between the low-level video features and the high-level 
query concepts can be so huge that the patterns defined by topics may be interpreted as a higher characterisation level and may help us to obtain a better retrieval performance. However, the most common ranking functions do not take into account the own nature of the topic space what eventually makes that many of them do not work properly in this representation.

\subsection{Objectives and structure}

The main objective of this work is to obtain an effective and efficient CBVR approach completely based on the rationale of latent topics in order to deal with the semantic gap challenge by means of the patterns defined by topics. First of all, the supervised Symmetric probabilistic Latent Semantic Analysis (sSpLSA) model is proposed to transform the classical retrieval approach into a class discovery problem what allows us to handle the user's searching concept as a mixture of hidden patterns. Subsequently, a new probabilistic ranking function is deduced from that model in order to estimate the probability that each sample of the database belongs to the query class (searching concept). Finally, the proposed retrieval approach is defined allowing both internal and external queries. In this work, we have considered a short-term RF approach, that is, each searching process is independent from one another. However, further improvements could be aimed at developing a long-term approach where the system learns from previous searches as well.

This work extends our previous work [23] where the sSpLSA model was introduced to obtain an initial ranking function which had some limitations. One of those limitations was assuming that queries are only from inside the database. There are two different ways the user can initialise a query, selecting samples from the own database or by providing external ones. In the 
first case, the user explores the database and selects some samples containing the concept of interest. However, that is not always the case. When the database is really huge or the query concept is very rare, it could be rather difficult to find proper samples to initialise the query. In those cases, it is more effective to initialise the query with external samples as long as the user has some examples of what they are looking for. In the present work, the retrieval model is extended and the ranking function is revised using more realistic assumptions what leads to an improvement of the retrieval

performance. In addition, this work extends the experimental part with a more comprehensive experimental setting, adding more relevant methods in the literature and using more databases.

The rest of the work is organised as follows: in Section 2, the proposed latent-topic retrieval model is presented including the definition of a new ranking function (Section 2.3) and a procedure (Section 2.4) to enable the use of external queries. Section 3 shows the retrieval experiments using three different databases: PAL [26], CCV [27] and TREVID [28]. Finally, Section 4 discusses the results and Section 5 draws the main conclusions arisen from the work.

\section{Probabilistic latent topic retrieval model}

\subsection{Probabilistic topic models}

In general, topic models are a kind of statistical graphical models which are able to uncover the hidden structure that pervade a data collection. Specifically, these methods take as an input a specific data probability matrix $P(W \mid D)$ which describes a corpus of documents $D=\left\{d_{1}, \ldots, d_{N}\right\}$ in a certain 
word space $W=\left\{w_{1}, . ., w_{M}\right\}$ and obtain as an output two probability matrices, the description of $K$ topics $Z=\left\{z_{1}, \ldots, z_{K}\right\}$ in words $P(W \mid Z)$ and the description of documents in topics $P(Z \mid D)$. The majority of topic methods are in the families of two models, probabilistic Latent Semantic Analysis (pLSA) [29] and Latent Dirichlet Allocation (LDA) [30]. Both pLSA and LDA are a reference in topic modelling although there are significant differences between them. On the one hand, pLSA uses the documents of the collection as parameters of the model what makes pLSA a high spatial demanding model and generates topic over-fitting when too many parameters are considered. On the other hand, LDA tries to overcome pLSA drawbacks by using two Dirichlet distributions, one to model documents $P(Z \mid D) \sim \operatorname{Dir}(\alpha)$ and another to model topics $P(W \mid Z) \sim \operatorname{Dir}(\beta)$. Logically, these parameters $\alpha$ and $\beta$ have to be estimated during the topic extraction process which adds an extra computational cost.

Despite the fact that the experimentation in [30] reveals that LDA is able to achieve lower perplexity than pLSA, it is not clear how the perplexity correlates with the performance in retrieval tasks. The same Blei in [31] concludes that pLSA often obtains a topic structure more correlated to the human judgement than LDA although the perplexity values suggest the opposite. In the standard LDA algorithm, the parameter estimation is performed by iterating over the document collection what produces that LDA requires a certain number of documents to adequately estimate its hyperparameters. In an application like CBVR, the concept to retrieve is a priori unknown because it is up to the user. Besides, the initialisation and feedback are often very limited. As a result, it is usual to deal with complex concepts 


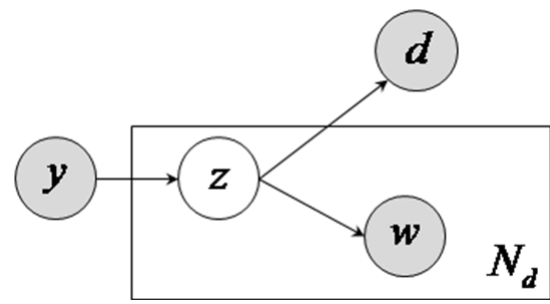

Figure 2: sSpLSA model. $y$ is the class, $z$ the topic (hidden variable), $w$ the word, $d$ the document and $N_{d}$ the number of words of $d$.

having very little information about them and in these circumstances pLSA is more accurate [32]. For these reasons, we have decided to use the pLSA model as the basis of our extended model for CBVR.

\subsection{Supervised symmetric probabilistic Latent Semantic Analysis (sSpLSA)}

The supervised Symmetric probabilistic Latent Semantic Analysis (sSpLSA) model (Figure 2) extends the unsupervised symetric pLSA [29] model by adding the observed random variable corresponding to class label $y$. In this case, the approach is directed to a similar scenario than the single-author topic model used by Fei-Fei and Perona [33] in the framework of a LDA-based model. The generative process of the sSpLSA model stems from the class probability distribution $p(y)$. In the model, classes $y$ are expressed as topic mixtures of topics $z$ according to parameters $p(z \mid y)$. Therefore, the process to generate a document $d$ can be interpreted as follows:

- A class $y$ is drawn for a document $d$ from the probability distribution $p(y)$.

- For each one of the $N_{d}$ words in the document $d$, 
- Given the document class $y$, a topic $z$ is chosen according to conditional distribution $p(z \mid y)$ that expresses classes in topics.

- Given the topic $z$ chosen, a word $w$ is drawn from the conditional distribution $p(w \mid z)$ that relates topics to words.

- Given the $N_{d}$ topics drawn to extract the words, a document $d$ is defined according to the class conditional distribution $p(d \mid z)$.

The sSpLSA model could be used to extract the topics of a data collection using information about class labels $(y)$ like a regular supervised topic model but it is not the goal here. We aim at relating the sSpLSA general model (Fig. 2) to the RF retrieval scheme (Fig. 1) in order to obtain a probabilistic ranking function based on sSpLSA. For that purpose, we use the following notation: $y^{\prime}$ is the query class and represents the kind of videos the user wants to extract from the database and $D^{\prime}=\left\{d_{1}^{\prime}, \ldots, d_{N^{\prime}}^{\prime}\right\}$ refers to the query set containing one or more positive examples of the query class. Note the difference between $y$ and $y^{\prime}$. The former $(y)$ is related to the general concept of class label information used in the sSpLSA model and the latter $\left(y^{\prime}\right)$ is the specific kind of videos the user wants to extract from the database in a specific retrieval session. Our objective is to sort the database using as a score of the ranking the probability that each document $d$ of the database belongs to the query class $y^{\prime}$, i.e. $p\left(y^{\prime} \mid d\right)$. In the next section, we are going to deduce the ranking function of the proposed approach deriving this probability over the sSpLSA model. 


\subsection{Latent Topic Ranking (LTR)}

Initially, we assume that a topic process has been carried out over the database in order to extract a specific number of topics $(K)$ and to express the whole collection according to those extracted topics as $P(Z \mid D)$. For the topic extraction task, it can be used either a supervised model (sLDA...) or unsupervised (LDA, pLSA...) one. It should be noted that in the supervised case topics are extracted using some initial class label information $y$ which does not have to be related to the concept $y^{\prime}$ (query class) that the user wants to retrieve in a particular session.

The proposed Latent Topic Ranking (LTR) function is aimed at providing a guess of the probability $p\left(y^{\prime} \mid d\right)$ that each document $d$ of the database belongs to the query class and using these probability values it performs the ranking at each retrieval iteration. According to the sSpLSA model (Fig. 2), this probability can be estimated from the present user's query by means of topic characterisations as follows. Let us express the conditional probability $p\left(y^{\prime} \mid d\right)$ by marginalising over topics:

$$
p\left(y^{\prime} \mid d\right)=\frac{p\left(y^{\prime}, d\right)}{p(d)}=\frac{\sum_{w} \sum_{z} p\left(w, d, z, y^{\prime}\right)}{p(d)}=\frac{\sum_{w} \sum_{z} p(w \mid z) p(d \mid z) p\left(z \mid y^{\prime}\right) p\left(y^{\prime}\right)}{p(d)}
$$

Where it has been assumed that the joint probability $p\left(w, d, z, y^{\prime}\right)$ is expressed according to the introduced sSpLSA model. Regarding the conditional topic probability of a given class $p\left(z \mid y^{\prime}\right)$, it can be estimated by marginalising over the query set $D^{\prime}=\left\{d_{1}^{\prime}, \ldots\right\}$ as follows: 


$$
p\left(z \mid y^{\prime}\right)=\sum_{d^{\prime}} p\left(z, d^{\prime} \mid y^{\prime}\right)=\sum_{d^{\prime}} \frac{p\left(z \mid d^{\prime}, y^{\prime}\right) p\left(d^{\prime}, y^{\prime}\right)}{p\left(y^{\prime}\right)}=\sum_{d^{\prime}} \frac{p\left(z \mid d^{\prime}, y^{\prime}\right) p\left(y^{\prime} \mid d^{\prime}\right) p\left(d^{\prime}\right)}{p\left(y^{\prime}\right)}
$$

Inserting (2) in (1) we obtain

$$
p\left(y^{\prime} \mid d\right)=\frac{\sum_{w} \sum_{z} p(w \mid z) p(d \mid z) \sum_{d^{\prime}} p\left(z \mid d^{\prime}, y^{\prime}\right) p\left(y^{\prime} \mid d^{\prime}\right) p\left(d^{\prime}\right)}{p(d)}
$$

The conditional probability $p\left(y^{\prime} \mid d^{\prime}\right)$ represents the probability that a document of the query belongs to the query class which is always true, therefore $p\left(y^{\prime} \mid d^{\prime}\right)=1$. Moreover, assuming the normalisation constraint over topics $\sum_{w} p(w \mid z)=1$, expression (3) can be simplified as follows:

$$
p\left(y^{\prime} \mid d\right)=\frac{\sum_{z} p(d \mid z) \sum_{d^{\prime}} p\left(z \mid d^{\prime}, y^{\prime}\right) p\left(d^{\prime}\right)}{p(d)}
$$

After multiplying and dividing by $p(z)$ and applying Bayes' rule $p(z \mid d)=$ $p(d \mid z) p(z) / p(d)$ we obtain

$$
p\left(y^{\prime} \mid d\right)=\sum_{z} \frac{p(d \mid z) p(z)}{p(d) p(z)} \sum_{d^{\prime}} p\left(z \mid d^{\prime}, y^{\prime}\right) p\left(d^{\prime}\right)=\sum_{z} \frac{p(z \mid d)}{p(z)} \sum_{d^{\prime}} p\left(z \mid d^{\prime}, y^{\prime}\right) p\left(d^{\prime}\right)
$$

Let us assume that the probability $p(d)$ of the documents of the database and the probability $p\left(d^{\prime}\right)$ of the documents of the query follows the same uniform distribution over the total number of documents of the database $|D|$, i.e. $p(d)=p\left(d^{\prime}\right)=1 /|D|$. This assumption implies that all the documents have the same prior probability independently of their number of words, features or even their relation with other samples. In the case of internal queries, 
it makes sense to use $1 /|D|$ as an estimation of $p\left(d^{\prime}\right)$ because queries are selected from the own database. Besides, even in the case of external queries the number of samples from outside the database is so reduced compared with the number of documents in the database $\left(\left|D^{\prime}\right|<<|D|\right)$ that the value $1 /|D|$ is a good approximation to $1 /\left(|D|+\left|D^{\prime}\right|\right)$. Thus, $p(z)$ can be estimated by marginalising the documents $d_{i}$ of the database and using Bayes' rule

$$
p(z)=\sum_{d_{i}} p\left(z, d_{i}\right)=\sum_{d_{i}} p\left(z \mid d_{i}\right) p\left(d_{i}\right) \approx \frac{1}{|D|} \sum_{d_{i}} p\left(z \mid d_{i}\right)
$$

Inserting (6) into (5), the probability $p\left(y^{\prime} \mid d\right)$ can be expressed as

$$
p\left(y^{\prime} \mid d\right) \approx \sum_{z} \frac{p(z \mid d)}{\sum_{d_{i}} p\left(z \mid d_{i}\right)}\left[\sum_{d^{\prime}} p\left(z \mid d^{\prime}, y^{\prime}\right)\right]
$$

In the present work, we have considered a short-term RF scheme what means that each retrieval session is independent from one another. In other words, all the information we have about the query class $y^{\prime}$ is provided by the samples of the query set $d^{\prime}$, therefore $y^{\prime} \approx d^{\prime}$ and then $p\left(z \mid d^{\prime}, y^{\prime}\right) \approx p\left(z \mid d^{\prime}\right)$. As a result, the final expression to estimate the probability $p\left(y^{\prime} \mid d\right)$ for the LTR function is as follows:

$$
p\left(y^{\prime} \mid d\right) \approx \sum_{z} \frac{p(z \mid d)}{\sum_{d_{i}} p\left(z \mid d_{i}\right)}\left[\sum_{d^{\prime}} p\left(z \mid d^{\prime}\right)\right]
$$

Expression (8) has two main factors. The left one is related to the document $d$ of the database we want to rank and the right factor represents the query at a specific stage of the retrieval process. In the first factor, $p(z \mid d)$ is learned off-line using any latent topic algorithm, for instance pLSA or LDA. Then, $\sum_{d_{i}} p\left(z \mid d_{i}\right)$ can be precomputed off-line as well using all the documents 
of the database. In the second factor, $p\left(z \mid d^{\prime}\right)$ is the probability that a given query document $d^{\prime}$ belongs to the topic $z$.

The ranking process is made as follows. First of all, $K$ topics are extracted from the database using some topic extraction method and subsequently each document $d$ of the database and the initial query documents $d^{\prime}$ are represented in these topics as $p(z \mid d)$ and $p\left(z \mid d^{\prime}\right)$ respectively. Later, the database is sorted according to the probability that documents $d$ belong to the query class $y^{\prime}$ using equation (8). Following the relevance feedback scheme, the $S$ most likely samples (scope) are showed to the user who selects the $P$ correctly retrieved samples. Then, these $P$ samples are used as feedback to expand the query. At each iteration, the query is expanded with more positive examples and probabilities $p\left(y^{\prime} \mid d\right)$ are recomputed to refine the ranking. In the end, the interactive process ends after $I$ iterations when the user has retrieved enough samples.

Comparing the LTR function (8) with the version in [23], we can observe two main differences. On the one hand, in LTR documents are normalised by the global use of the topics in the collection, therefore the least used topics are able to generate a higher probability values. That is, the match with the query patterns is calculated by fostering the least used topics. On the other hand, expression (8) uses $p\left(z \mid d^{\prime}\right)$ (query expressed in topics) instead of $p\left(d^{\prime} \mid z\right)$ (topics expressed in query documents). This allows to get rid of the simplification we made in the ranking process of [23] where we assumed that topics do not depend on queries to approximate $p\left(d^{\prime} \mid z\right)$ as the transposed and normalised version of $p\left(z \mid d^{\prime}\right)$ what is not a real premise.

Another important change is based on allowing the use of internal and 


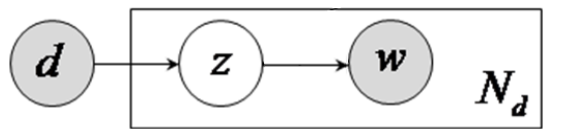

Figure 3: Graphical model representation of the aspect model in the asymmetric pLSA parametrization used by Hofmann in [29]. $d$ is the document, $z$ the topic (hidden variable), $w$ the word and $N_{d}$ the number of words of $d$.

external query samples. The off-line topic learning process obtains $P(W \mid Z)$ and $P(Z \mid D)$ from the database. Thus, when queries are inside the database, we already have the description of the query documents in topics. However, when queries are initialised with external samples, we have to use an estimation procedure to represent those external documents in the previously extracted topics. The following section shows the used procedure to represent external samples in a set of given topics.

\subsection{Expectation Maximisation eStimator (EMS)}

As it was mentioned earlier, regular topic algorithms such as pLSA and LDA are able to obtain from a data collection the description of topics in words as $P(W \mid Z)$ and the representation of the database in topics as $P(Z \mid D)$. However, in this work queries can be initialised with samples from outside the database and therefore the proposed approach requires an additional procedure to represent external query documents $D_{\text {out }}^{\prime}=\left\{d_{\text {out }}^{\prime}, \ldots\right\}$ in a given set of topics as $P\left(Z \mid D_{\text {out }}^{\prime}\right)$. Following the same notation than before, the upper-case letter represents the set and the lower-case an instance of that set.

We use the asymmetric version pLSA model (Fig. 3) to define the Expectation Maximization eStimator (EMS) procedure. Specifically, the pa- 
rameter $p\left(z \mid d_{\text {out }}^{\prime}\right)$, which represents an external query document in a given set of topics, can be estimated following the pLSA model by maximizing the log-likelihood using the Expectation-Maximization (EM) algorithm. Let us define the joint distribution of the model Eq. (9) and the log-likelihood Eq. (10) in terms of the joint probability distribution

$$
\begin{array}{r}
p\left(w, z, d_{\text {out }}^{\prime}\right)=p(w \mid z) p\left(z \mid d_{\text {out }}^{\prime}\right) p\left(d_{\text {out }}^{\prime}\right) \\
\mathcal{L}=\sum_{w} n\left(w, d_{\text {out }}^{\prime}\right) \log \left[p\left(w, d_{\text {out }}^{\prime}\right)\right]
\end{array}
$$

Where $n\left(w, d_{\text {out }}^{\prime}\right)$ is the number of occurrences of the word $w$ in the document $d_{\text {out }}^{\prime}$. In order to maximize the log-likelihood by EM, the complete log-likelihood can be expressed using the latent variables $z$ as

$$
E=\sum_{w} n\left(w, d_{\text {out }}^{\prime}\right)\left(\sum_{z} p\left(z \mid w, d_{\text {out }}^{\prime}\right) \log \left[p(w \mid z) p\left(z \mid d_{\text {out }}^{\prime}\right) p\left(d_{\text {out }}^{\prime}\right)\right]\right)
$$

Introducing in expression (11) the normalisation constraints of the parameter $p\left(z \mid d_{\text {out }}^{\prime}\right)$ by inserting the appropriate Lagrange multiplier $\beta$ :

$$
H=E+\beta\left[1-\sum_{z} p\left(z \mid d_{\text {out }}^{\prime}\right)\right]
$$

Taking the derivative with respect to $p\left(z \mid d_{\text {out }}^{\prime}\right)$, setting the expression equal to zero and solving the equation to isolate the parameter, the M-step of the EM algorithm is expressed as

$$
p\left(z \mid d_{\text {out }}^{\prime}\right)=\frac{\sum_{w} n\left(w, d_{\text {out }}^{\prime}\right) p\left(z \mid w, d_{\text {out }}^{\prime}\right)}{\sum_{z} \sum_{w} n\left(w, d_{\text {out }}^{\prime}\right) p\left(z \mid w, d_{\text {out }}^{\prime}\right)}
$$



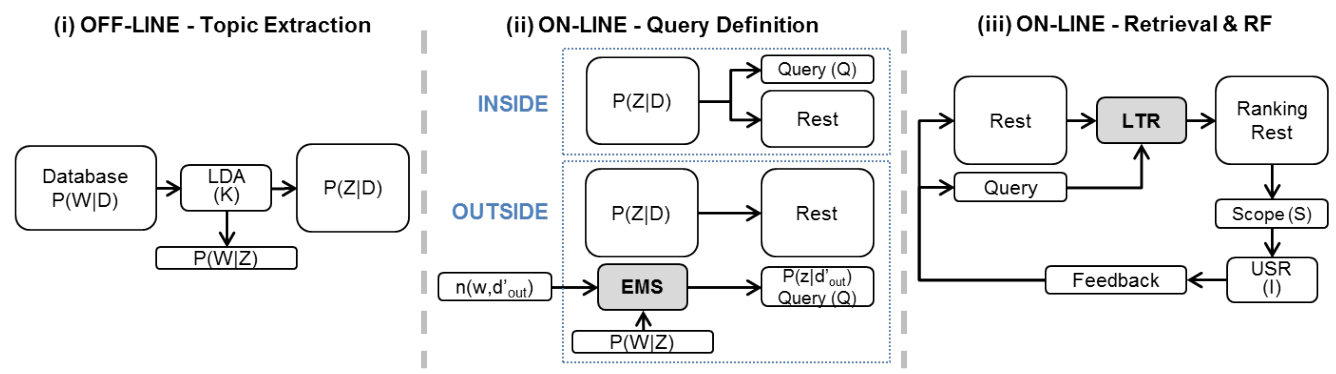

Figure 4: Proposed approach scheme. $K$ (number of topics), $Q$ (number of samples to initialise the query), $I$ (number of feedback iterations) and $S$ (number of top ranked samples).

For the E-step, we need to estimate the parameter $p\left(z \mid w, d_{\text {out }}^{\prime}\right)$. Applying the Bayes' rule and the chain rule we obtain

$$
p\left(z \mid w, d_{\text {out }}^{\prime}\right)=\frac{p\left(w, z, d_{\text {out }}^{\prime}\right)}{p\left(w, d_{\text {out }}^{\prime}\right)}=\frac{p(w \mid z) p\left(z \mid d_{\text {out }}^{\prime}\right)}{\sum_{z} p(w \mid z) p\left(z \mid d_{\text {out }}^{\prime}\right)}
$$

The EM process is performed as follows. First of all, the external query document $n\left(w, d_{\text {out }}^{\prime}\right)$ and the set of previous topics $p(w \mid z)$ are loaded. Secondly, $p\left(z \mid d_{\text {out }}^{\prime}\right)$ is randomly initialised. Then, the E-step Eq. (14) and the M-step Eq. (13) are alternated until a convergence condition is reached. As default settings, we have used a threshold of $10^{-6}$ in the difference of the log-likelihood Eq. (10) between two consecutive iterations and a maximum of 1000 EM iterations to assure a fixed and sensible computational cost in the convergence process.

\subsection{Latent Topic-based Relevance Feedback Framework}

The proposed retrieval approach is made up of three main phases (Fig. 4): (i) off-line topic extraction, (ii) on-line query definition and (iii) on- 
line retrieval and relevance feedback. In the first phase (i), the LDA [30] algorithm is used over the collection in order to extract $K$ topics as $P(W \mid Z)$ and to represent the samples of the database in those topics as $P(Z \mid D)$. Note that $P(W \mid D)$ represents the normalised word count of the documents of the collection. We have selected LDA instead of pLSA because the spatial cost of pLSA for the tested collections is unaffordable, however pLSA or any other topic model can be used in this phase instead. Once this off-line process has been carried out the system changes to the on-line mode which contains two more phases.

The phase (ii) on-line query definition is corresponded to the Stage 0 of the RF scheme showed in the Fig. 1. In this part, the user has two different alternatives to initialise the query. When queries are from inside the database, $Q$ query samples are selected from the own collection as the initial query set and the rest of the samples make the Rest set which is the basis to perform the ranking. When queries are from outside, the EMS function is used to represent the external query samples $n\left(w, d_{\text {out }}^{\prime}\right)$ in the previous $K$ extracted topics $P(W \mid Z)$. Note that $n\left(w, d_{\text {out }}^{\prime}\right)$ represents the word count of the external query documents. Then, these external samples expressed in topics $p\left(z \mid d_{\text {out }}^{\prime}\right)$ make the Query set and the whole database $P(Z \mid D)$ is used as the Rest set. The external samples have to be represented in the same initial characterisation space $W$ than the database used to extract the topics e.i. using the same descriptor.

Once the Query and Rest sets are initialised, the proposed approach changes to the phase (iii) on-line retrieval and relevance feedback which represents the stages 1 and 2 of Fig. 1. In this iterative stage, the LTR function 
uses both the Query and Rest sets to obtain a ranking of Rest by using equation (8). From this ranking, the $S$ top samples are shown to the user who selects the positive samples to provide the feedback. These correctly retrieved samples are used to expand the Query and besides they are removed from the Rest set. In order to reduce the complexity of the interaction process, only positive feedback samples are used to expand the query. Finally, with the updated Query and Rest sets the next iteration is triggered. The number of total feedback iterations $I$ depends on the user, that is, the user decides when the interaction ends.

\section{Experiments}

This section presents the experimental part of the work. Section 3.1 describes the kind of retrieval simulations performed in the experiments and the retrieval methods of the literature used to test the proposed approach. Subsequently, sections 3.2, 3.3 and 3.4 show the retrieval results for three different databases: PAL [26], CCV [27] and TRECVID 2007 [28].

\subsection{Short-term Relevance Feedback simulations}

A total of six different user interaction scenarios are defined to evaluate the effectiveness of the proposed approach with respect to seven different retrieval methods over three databases. We assume that each database used for the simulations is a pre-labelled collection, i.e. it is annotated according to a specific set of classes, and besides it is partitioned in two balanced halves, training and test. 


\subsubsection{Parameters of the simulations}

Following the scheme of the proposed approach (Figure 4), the on-line stage has three main parameters: $Q$ the number of samples of the initial query, $S$ the number of top examined items and $I$ the number of total iterations. The target of each simulation is directed to retrieve samples of a specific class, but without using any class label information. In other words, the query is initialised with $Q$ samples of a single class $c$ and the simulation process has to retrieve samples of that class through $I$ feedback iterations. At each iteration, the $S$ top ranked items are inspected by a simulated user who marks the samples of the class $c$ (positive samples). These positive samples are computed as correctly retrieved samples and they are used to expand the query. Finally, the expanded query is triggered as a new query for the next iteration.

In this work, we assume a simulated-user reliability of a 100\% in order to simplify, but some uncertainty could be introduced in the simulation process. This uncertainty could be introduced into the retrieval system in a soft way or in a more intense way. An example of the former case could be by limiting the number of feedback examples per iteration. That is, instead of selecting all the positive examples each feedback iteration just marking a few correctly retrieved samples. Note that, this is a quite common situation because real users do not often analyse the whole content of a screen. Another example of a more aggressive uncertainty could be by introducing some mistakes in the feedback process. This fact may produce a remarkable precision drop and its study would be interesting to test the stability of the different retrieval methods. 
The experiments are divided in two kinds of simulations according to the initialisation of the query (Fig. 4): (a) when queries are from inside the database and (b) when queries are from outside the database. In the first case (a), the complete dataset is used to extract $K$ topics by LDA and then for each class $c$ of the database queries are initialised with $Q$ random samples of the that class. This random initialisation is repeated $R$ times in order to obtain an average value of the retrieval precision and an average computational time per query. Note that by complete dataset we mean the union of both partitions training and test because we assume that the dataset is initially divided into these two balanced partitions.

When queries are from outside the database (b), the training partition is used to extract $K$ topics using LDA and the test set is represented according to those topics by means of the EMS function. Then, each sample of the test set is used to trigger an external query and therefore the target is to retrieve videos from the training set which belong to the same class as the query sample of test set. Note that in this case there is no point in considering the parameter $R$ because each test sample is a query itself, thus there is not random initialisation. Like in the previous case, the performance measures of the simulations are the average precision and average computational time per query.

Table 1 shows the six different simulations considered for the experiments. Those parameters have been set taking into account the user comfort in the retrieval process. For real users, it is not comfortable to initialise the query with many samples and for that reason we assume that the user only provides one or two examples, that is, $Q=\{1,2\}$. The number of feedback iterations 
Table 1: Parameters of the simulations for the experiments.

\begin{tabular}{|c|c|c|c|c|c|c|c|}
\hline \multicolumn{4}{|c|}{ (a) INSIDE } & \multicolumn{4}{c|}{ (b) OUTSIDE } \\
\cline { 1 - 4 } Simulation & Q & I & S & Simulation & Q & I & S \\
\hline 1 & 1 & 5 & 20 & & & & \\
\cline { 1 - 4 } 2 & 2 & 5 & 20 & 1 & 1 & 5 & 20 \\
\hline 3 & 1 & 5 & 40 & & & & \\
\cline { 1 - 4 } 4 & 2 & 5 & 40 & & 1 & 5 & 40 \\
\hline
\end{tabular}

is another important parameter. The retrieval systems require a certain number of iterations to be properly aided, but a high number of iterations affect negatively to the user's attention. Therefore, we consider $I=\{5\}$ to balance the efficacy of the retrieval system and the user's preferences.

Regarding the scope $S$, somehow this parameter is related to $I$. A bigger scope may reduce the number of feedback iterations, but it makes users to check more samples at each iteration which eventually affects to their comfort. As a result, we have chosen two reasonable values for the scope, $S=\{20,40\}$. Considering that the average number of videos which can be shown in a regular screen is around 20, that configuration simulates two different scenarios: one where the simulated users are inspecting only the first screen at each feedback iteration and another where they are inspecting two screens per iteration.

Other important parameters are $R$, the number of times the query is randomly initialised, and $K$, the number of extracted topics. Note that those parameters change from database to database, therefore they are not included in table 1 but in the tables with the results for each database in Section 3.5. The parameter $R$ has been selected to perform a reasonable number of ran- 
dom initialisations of the query to obtain robust average values. Regarding the number of topics, selecting the right number of topics is an open-ended issue, especially in the visual domain. In the literature, there are several approaches which try to tackle this problem but all of them require performing the topic extraction process several times which makes them impractical to be used in a real system. As a result, we have tested different number of topics according to the size of the databases to make the results consistent.

\subsubsection{Retrieval methods for comparison}

In order to evaluate the proposed approach, we have compared our method with seven different ranking functions. These functions have been selected because they are widely used in literature and they usually obtain a good performance in retrieval or classification tasks. In this work, we have used a short-term Relevance Feedback approach, thus simulations do not use training information of previous searches. Other important retrieval approaches need search examples as training set. Ranking SVM [34] is a powerful tool for optimising the similarity function of content-based retrieval systems, but it needs a reasonable training set to carry out the ranking. In the experimental comparison, we have only used retrieval methods suitable for a short-term Relevant Feedback scheme like the proposed approach. Specifically, we have considered distance-based and transductive-based ranking methods for the experiments.

The following distance/similarity ranking functions [35] have been tested: Euclidean distance (EC), symmetric Kullback-Leibler divergence (KL), Cosine similarity (CS), Hellinger distance (HL) and Bhattacharyya distance (BC). These functions have been used on the original BoW representation 
of the dataset $P(W \mid D)$ and besides on the topic space generated by LDA $P(Z \mid D)$ in order to compare the retrieval performance in both cases. The distance/similarity based ranking sorts the samples of the database according to the minimum distance or maximum similarity to the query. In the case that the query has more than one sample we have computed the arithmetic mean of the measure. Specifically, we have chosen this averaging strategy rather than a max pooling one because in CBVR the user's initialisation and feedback are too limited to take advantage of sub-sampling the query set.

Regarding the transductive learning, we have selected MR (Manifold Ranking [13]) and LRGA (Local Regression and Global Alignment [14]) as two of the most important retrieval algorithms. However, LRGA suffers from a high computational cost when it is used over a large number of samples with high dimensionality. For this reason, we found that LRGA was not computationally affordable for the considered databases and therefore we have only tested MR ranking in both spaces $P(W \mid D)$ and $P(Z \mid D)$.

Additionally, we have tested another method in $P(Z \mid D)$. Zhang et al. [15] presented an image retrieval system which uses the cosine similarity function in the topic space to rank the database. It uses positive and negative samples in the Relevance Feedback (RF) process and summarises the query set in only one sample in the initial representation space $P(W \mid D)$. Then, this sample is represented as $p(z \mid d)$ according to the topics extracted from the database and eventually the cosine similarity is computed in the topic space to perform the ranking. For comparison purposes, we have adapted this approach to the framework used in this work in order to deal with only positive feedback. Algorithm 1 shows the Zhang (ZH) ranking function adapted for 
the experiments.

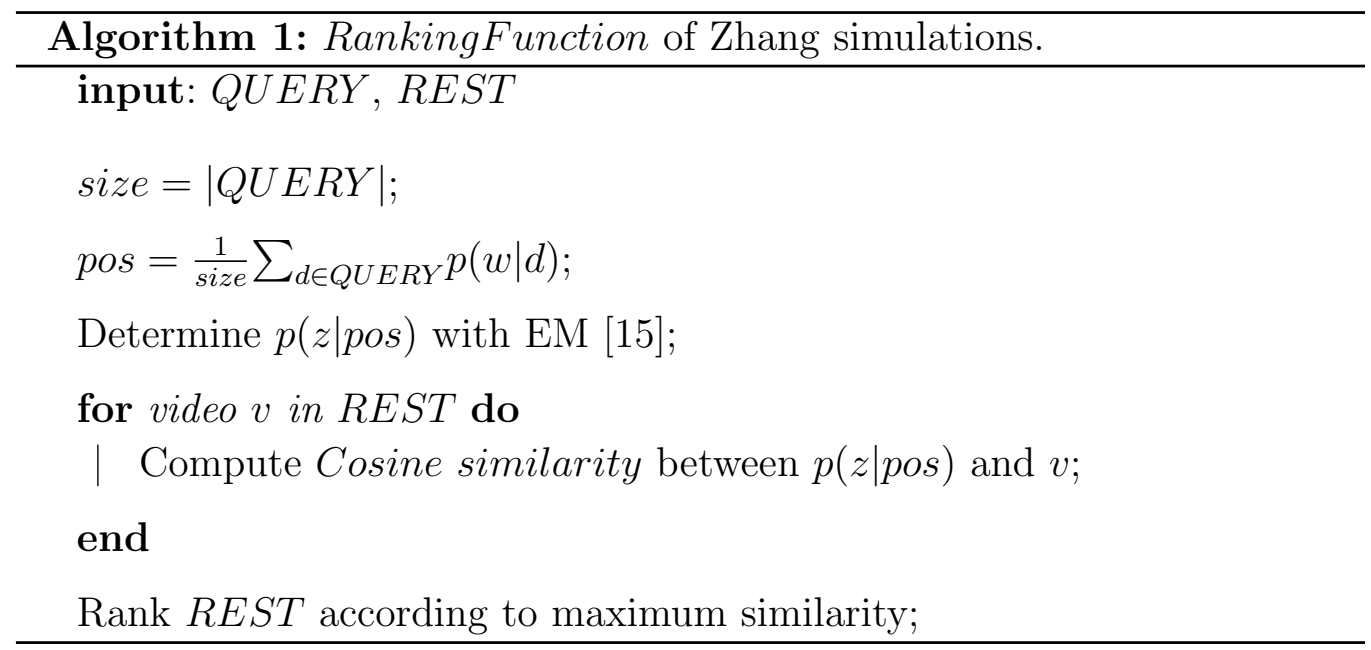

\subsection{Productive Ageing Lab (PAL) database}

The Productive Ageing Lab (PAL) face collection [26] contains 573 colour images of size $640 \times 480$ pixels corresponding to 223 males and 350 females with ages ranging from 18 to 93. The dataset has been randomly split into two balanced partitions, one for training with 112 males and 175 females and another for test with 111 males and 175 females. As a characterisation of the data, we have used the images converted into grey levels, scaled to $16 \times 13$ pixels and vectorised. As a result, the original feature space $P(W \mid D)$ of this database contains 208 words.

We first use this dataset to find out easily the differences among the output rankings obtained by the tested retrieval methods. Gender recognition is a 2-class problem with a wide intra-class variety, i.e. two very different faces could belong to the same gender, and this fact may easy the task to detect small ranking differences. Note that the gender recognition problem 
is extensively studied in the literature and the objective here is not to obtain a good accuracy but to compare the different output rankings.

\subsection{Columbia Consumer Video (CCV) database}

The Columbia Consumer Video (CCV) dataset [27] contains 9317 YouTube videos over 20 semantic categories, most of which are complex events, along with several objects and scenes. The authors of the database provide two balanced partitions, one for training with 4659 samples and another for test with 4658 samples. Besides, they provide three different video descriptors SIFT, STIP and MFCC. For the experiments, we have used the characterisation based on the SIFT descriptor which contains 5000 words. In particular, this codification is made up of the concatenation of five different parts: (1) the complete sample and (2)-(5) the division of the sample in a $2 \times 2$ grid. Each one of these parts is encoded using 1000 words as the concatenation of two different vocabularies: (a) BoW with 500 clusters over SIFT descriptor and Hessian-Affine detector and (b) BoW with 500 clusters over SIFT descriptor and DoG detector.

In this corpus, we have detected some samples with null descriptor content and others without annotation. In both cases these samples have been removed for the experiments. For the remaining ones, those samples labelled with more than one category have been replicated one for each class. As a result, we have considered a total of 7846 video samples, 3914 of training and 3932 of test, annotated in 20 classes as it is shown in Fig.5. 


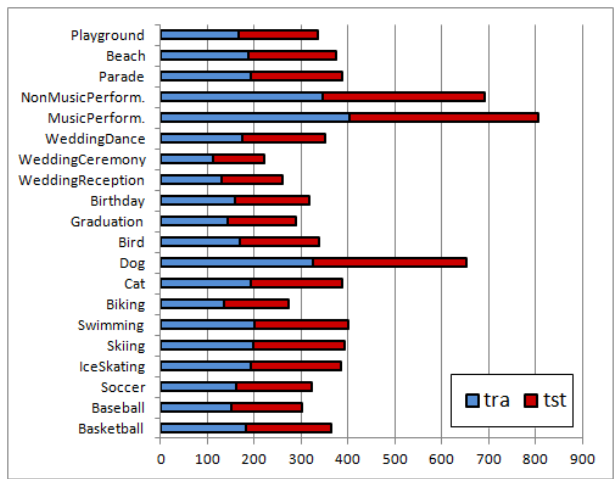

Figure 5: Samples per class of the CCV database.

\subsection{TRECVID 2007 database}

The TRECVID 2007 collection [28] is made up of 47,548 video shots which are annotated according to 36 semantic concepts. These categories were selected in TRECVID 2007 evaluation and they include several objects as well as complex events and scenes. Regarding the description of the database, we have used a similar characterisation than in the case of CCV. Specifically, we have followed the suggestions of van de Sande et al. [36] about using opponent SIFT histograms when choosing a single descriptor and no prior knowledge about the dataset is considered. The software provided by van de Sande has been applied to the middle frame of each shot and each sample has been encoded using a 3-level spatial pyramid codebook $(1 \times 1,2 \times 2$ and $4 \times 4$ ) what makes a total of 2688 words per shot. In order to make affordable the computational cost of the topic extraction task, we have reduced the original database by selecting a balanced subset with a similar size to the CCV collection. Specifically, we have divided the whole collection in 10 balanced partitions. Later, we have removed the classes under 100 samples in any partition, resulting a total of 17 selected classes. Finally, we have 


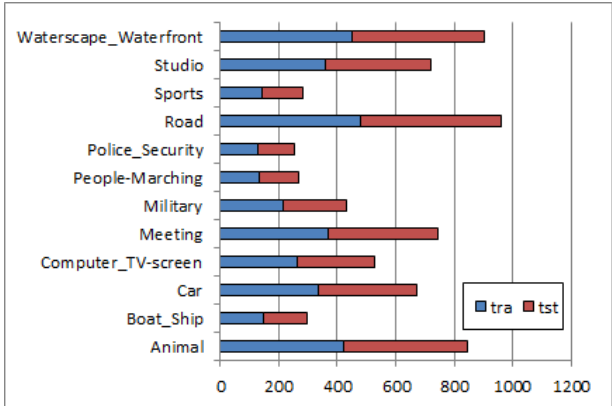

Figure 6: Samples per class of the considered subset of TRECVID 2007.

chosen one random partition as a training set and another as a test. Figure 6 shows the considered subset of 8974 samples with 4487 for training and 4487 for test annotated in 17 classes.

\subsection{Results}

Tables 2, 3 and 4 present the retrieval result in terms of Average Precision (AP) and average computational Time per query in seconds (T) running in a single processor Intel Xeon E5-2640. Each table corresponds to a particular database and the way they are organised is the following. In columns we have the six different simulations described in section 3.1, the first four (a) using internal queries and the last two (b) with external ones. The parameters of each simulation $(R, Q, I, S)$ are indicated in the headings of the columns. In rows we have the different retrieval methods used for the experiments. In particular, there are three groups: LTR which contains the results of the proposed approach using several number of topics $(K), P(W \mid D)$ which has the results of six different ranking functions in the original characterisation space and $P(Z \mid D)$ contains the results of seven different ranking functions in the best topic space among the tested number of topics. 
Related to the ranking functions, we use the following terminology: Euclidean distance (EC), symmetric Kullback-Leibler divergence (KL), Cosine similarity (CS), Hellinger distance (HL), Bhattacharyya distance (BC), Manifold ranking [13] (MF) and Zhang approach [15] (ZH).

\section{Discussion}

The first noteworthy point is the remarkable precision gains provided by topic models in the performed retrieval simulations. Comparing the best average precision value obtained in the original BoW space $P(W \mid D)$ with the best value among the seven ranking functions tested in the topic space $P(Z \mid D)$, we observe that in the topic space the precision is increased on average a $20.35 \%$ for the PAL database, $67.14 \%$ in the case of CCV and $21.10 \%$ for TRECVID. These significant precision gains support our statement that the hidden patterns provided by topic models are useful to fill the semantic gap in CBVR. Topic models have shown to help in many areas, such us text categorisation or image recognition, but in tasks where precision is important, like in CBVR, they have been traditionally considered useless. Some authors have this belief because the best ranking functions in the original BoW space tend not to work properly in the latent space. As we can see in the results, the best ranking functions in the original BoW space are EC (for PAL) and MF (for CCV and TREVID) but these two functions are often two of the worse in the latent space. However, the CS function is able to obtain a real precision improvement in the topic space. In fact, CS is the unique function which has shown to be effective in the latent space among the tested retrieval methods of the literature. 
Table 2: Retrieval result for PAL database: Average Precision (AP) and average seconds per query $(\mathrm{T})$. For each group of ranking functions (in rows), the best AP value of each simulation is highlighted in bold and the best global value among all methods is underlined.

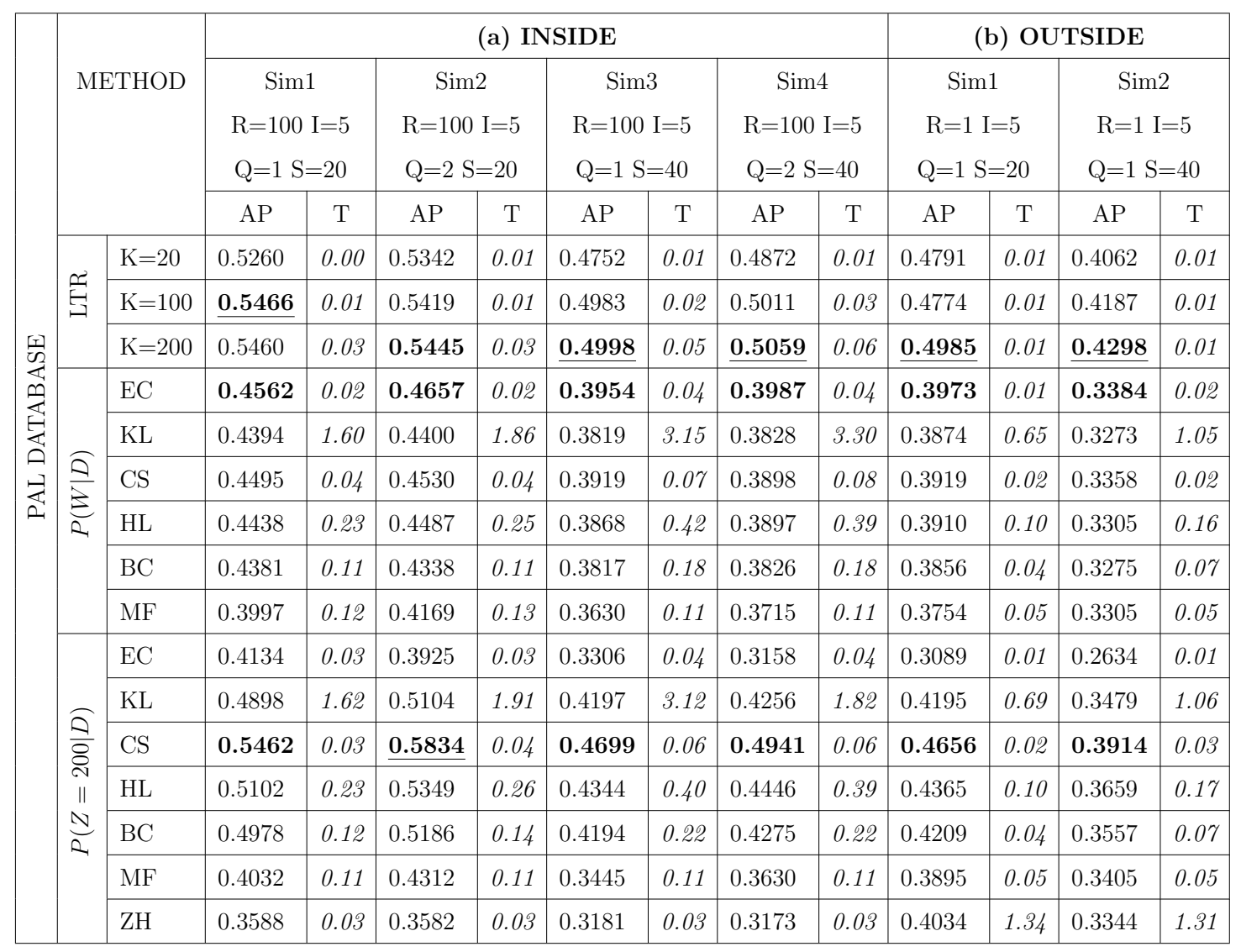


Table 3: Retrieval result for CCV database: Average Precision (AP) and average seconds per query (T). For each group of ranking functions (in rows), the best AP value of each simulation is highlighted in bold and the best global value among all methods is underlined.

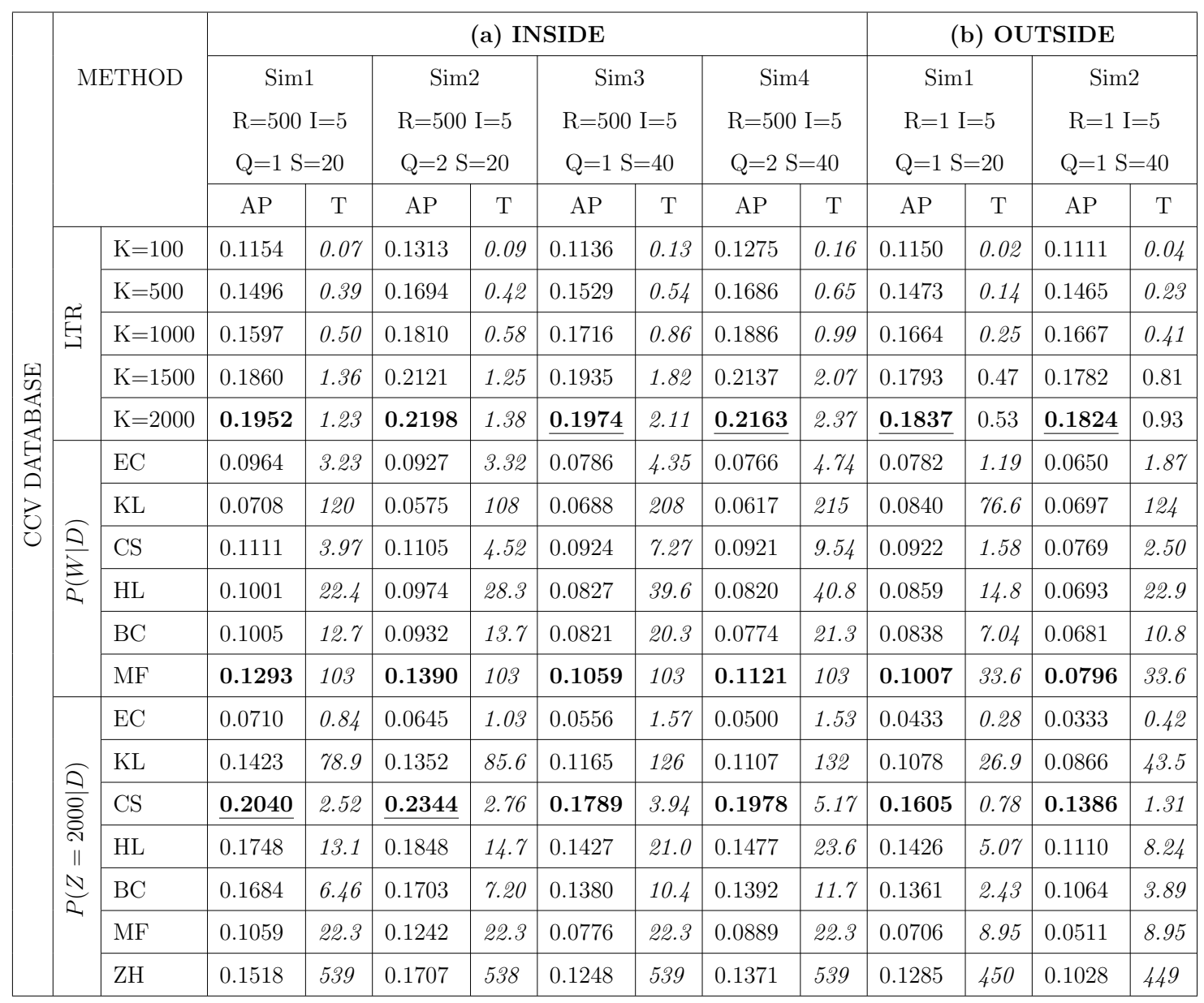


Table 4: Retrieval result for TRECVID database: Average Precision (AP) and average seconds per query (T). For each group of ranking functions (in rows), the best AP value of each simulation is highlighted in bold and the best global value among all methods is underlined.

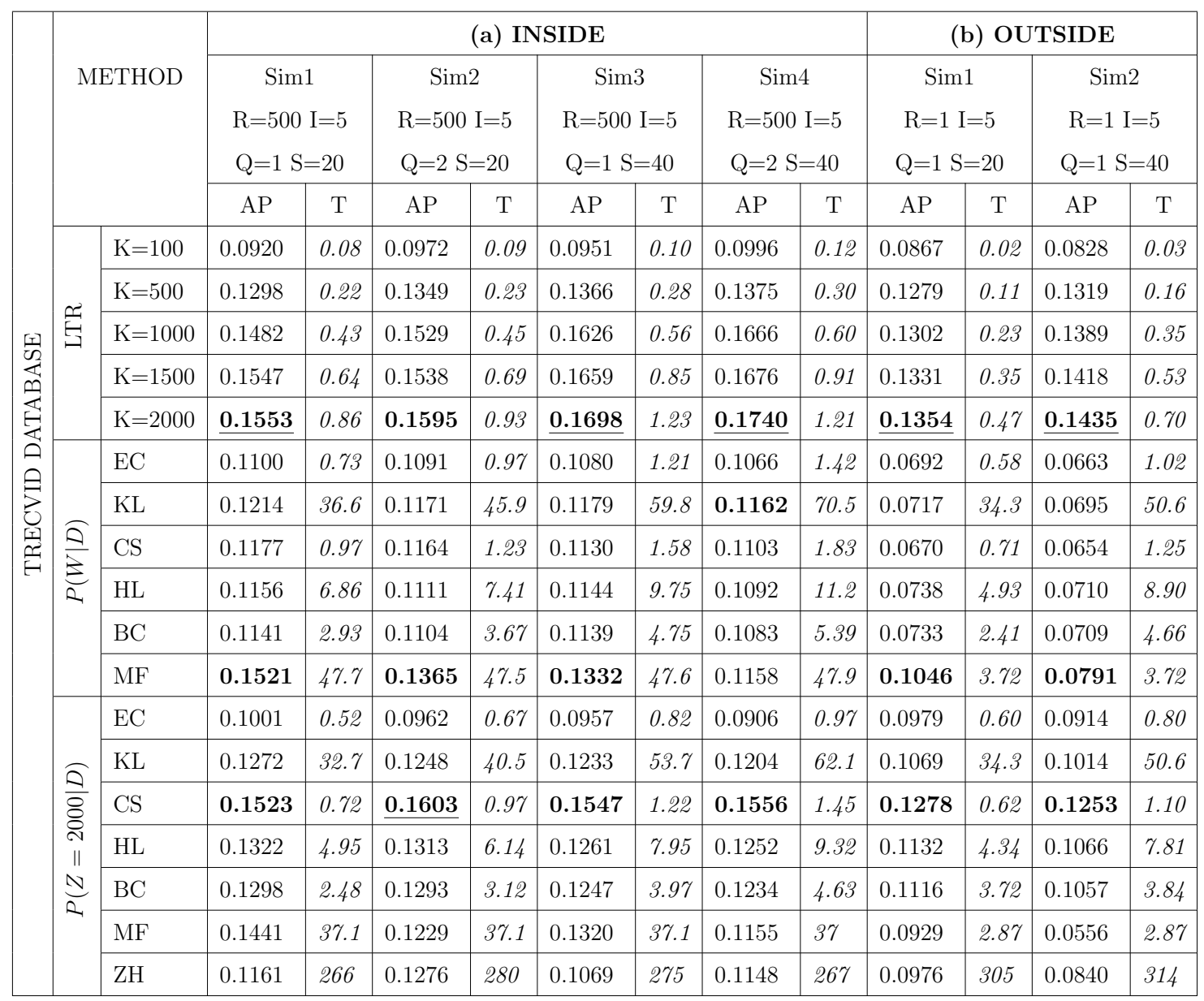


Another noticeable question related to the latent space is the adequate number of topics. We have tested several values for each database and the best precision results are obtained using the highest numbers, that is, 200 topics for PAL database and 2000 for both CCV and TRECVID. However, for PAL and TRECVID the precision improvement using these values is quite slight compared with the results obtained with 100 and 1500 respectively. This indicates that, depending on the database and the kind of queries, increasing the number of topics reaches a point in which it does not provide an actual improvement. Selecting the appropriate number of topics is an important question and still remains an open-ended issue in the literature. Even though the number of topics may significantly affect to the performance of a system, for this kind of application it is more important to have enough topics to obtain a fine granularity of patterns to describe queries than to find out exactly the optimum number. Somehow, it is similar to the case of finding the optimum number of clusters in a classification problem. As long as you have enough clusters to represent the classes it is not so important if some classes are represented with more than exactly one cluster.

In general, the results show a similar trend in the three tested databases. The LTR function achieves the best retrieval precision on average compared with the best methods in both the original BoW space $P(W \mid D)$ and the latent space $P(Z \mid D)$. In the PAL database, LTR outperforms EC-pwd in a $23.38 \%$ and CS-pzd in a 2.52\%. For CCV, the precision gain of LTR is a 79.19\% over MF-pwd and a 7.22\% over CS-pzd. In the case of TRECVID, LTR increases the precision of MF-pwd in a $29.58 \%$ and the precision of CS-pzd in a $7.00 \%$. 
Related to the parameters of the simulations, we observe the that average precision tend to increase using a bigger $Q$ (number of samples to initialise queries) and it drops with a larger value of $S$ (scope). The rationale behind this is the following: on the one hand, initialising queries with more samples provides more information about the concept of interest and then the retrieval systems is more effective. On the other hand, considering a larger scope makes the retrieval system use more samples which are less likely to belong to the query class what eventually generates a precision drop.

According to the results, the proposed LTR shows a good robustness regarding the parameters $Q$ and $S$ of the simulations. Focusing on CCV and TRECVID, the proposed LTR method obtains a similar precision gain to the best tested function (CS-pzd) when the parameter $Q$ increases (Sim2inside and Sim4-inside). In addition, LTR is able to reduce the precision drop compared with CS-pzd when the parameter $S$ is increased (Sim3-inside, Sim4-inside and Sim2-outside).

The proposed LTR function is able to outperform the tested methods in the original BoW space and in the latent space with the exception of CS-pzd. For that reason, we discuss in more detail the differences between LTR and CS to highlight the advantages of the proposed approach.

\subsection{Cosine Similarity (CS) vs. Latent Topic Ranking (LTR)}

The CS function uses the cosine of the angle between two samples as a similarity measure. That is, the most similar documents to the query are those which have the lowest angle with respect to the query (angular similarity). In the case the query has more than one document, we have used the average cosine similarity value. Equation (15) shows the CS function 
where $d$ represents a document of the database and $D^{\prime}$ the query set.

$$
\operatorname{Sim}\left(d, D^{\prime}\right)=\frac{\sum_{d^{\prime} \in D^{\prime}} \cos \left(\theta_{d, d^{\prime}}\right)}{\left|D^{\prime}\right|}=\frac{\sum_{d^{\prime} \in D^{\prime}} \frac{d \cdot d^{\prime}}{|d|\left|d^{\prime}\right|}}{\left|D^{\prime}\right|}
$$

The proposed LTR function provides a probabilistic approach to discover the most likely samples according to the query. As it is shown in equation (16), this function can be interpreted as a weighted scalar product between the document $d$ and a summary of the query set in a single document. The topics weights are computed as the inverse of the prior of each topic in the database. Therefore, the least used topics generate higher probability values, that is, the LTR function is a weighted scalar product which fosters the least used topics in the database. Intuitively, this makes sense because the least used patterns may allow us to discriminate better among samples for complex query concepts.

$$
\operatorname{LTR}\left(d, D^{\prime}\right)=\sum_{z} \underbrace{\left(\frac{1}{\sum_{d_{i}} p\left(z \mid d_{i}\right)}\right)}_{\text {topic weight }} \overbrace{\underbrace{(p(z \mid d))}_{\text {document }} \underbrace{\left(\sum_{d^{\prime} \in D^{\prime}} p\left(z \mid d^{\prime}\right)\right)}_{\text {query summary }}}^{\text {weighted scalar product }}
$$

At the same time, the scalar product (dot product) between two vectors is directly proportional to the projection of the first on the second vector. That is, $\left(d \cdot d^{\prime}\right)=\left|d^{\prime}\right|$ Projection $_{d-d^{\prime}}$. Logically, cosine similarity and LTR function have some similar features because the less angle often implies the more projection and then the more scalar product value. However, there is a main difference which enables the LTR function to overcome the cosine similarity retrieval precision. The weighting scheme gives more flexibility to 


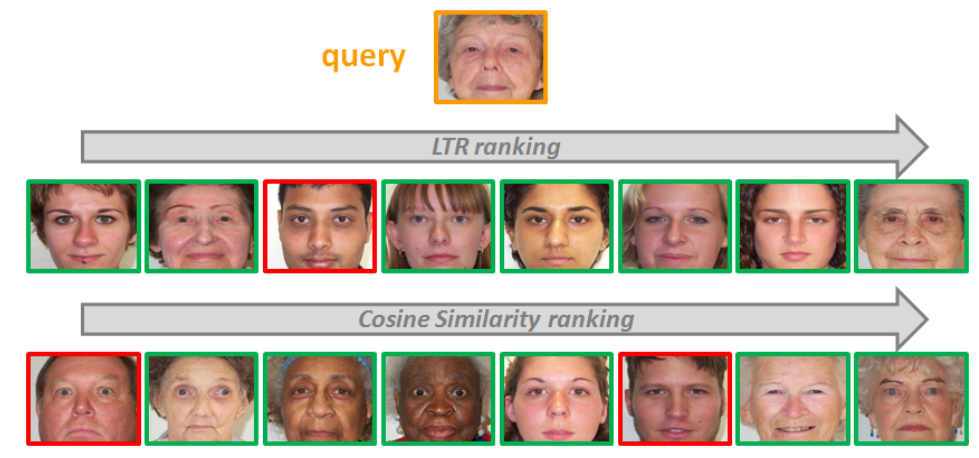

Figure 7: Example of gender retrieval. Cosine and LTR rankings at the first iteration with a scope of 20 . The figure only shows the images which are different in both 20-top rankings ( 8 pictures). The images are sorted from left to right according to the original 20-top ranking order.

the LTR function in order to deal with the semantic gap challenge.

Comparing both behaviours, the LTR function selects the documents within a margin of larger weighted scalar projection by fostering the least used topics. In a real application, this produces a top ranking with more variety of documents and then the user's feedback is able to provide more relevant information about the scope of the query concept. Let us see it through an example of gender retrieval using the PAL database. We are going to use an initial query of an elderly woman to compare the LTR and CS rankings at the first ranking iteration. Assuming queries from inside the PAL database, Figure 7 shows the differences between both 20-top rankings at the first iteration. The shared images have been removed to highlight the differences between the two ranking functions.

First of all, it should be noted the relationship between LTR and CS. A total of 12 images are the same in both 20-top rankings at the first iteration. Specifically, 8 white old women, 2 black women, one black man and 
another white man are shared by both rankings. This fact clearly shows the aforementioned relation between projection (LTR) and angular similarity (CS). However, we can appreciate a very important difference between the not overlapped images of both rankings. The cosine similarity function tends to retrieve samples of older women (the initial query) whereas LTR first retrieves women with different appearances. That is, the LTR function provides a broader kind of women images, thus the proposed approach is able to obtain a broader and more meaningful feedback about the query class.

As it was introduced in Section 1, the main problem in CBVR is the semantic gap challenge i.e. the difference between the user's understanding and the data representation. In CBVR, the same video sample can be related to very different concepts (queries) and the only way we have to distinguish among them is by the user's feedback. Therefore, enriching the query with a wide variety of positive examples in the feedback is a key factor to deal with unconstrained concepts.

Figure 8 shows the number of correctly retrieved videos per ranking iteration for the experiments using the CCV database. In both internal and external queries, we can see how the CS function archives the best performance at the first iteration and then the precision decreases in the subsequent iterations. However, LTR obtains the best performance at the second iteration after the first user's feedback and for the following iterations the precision drop is smoother than in the case of CS. This example shows that the feedback extracted from the LTR ranking contains a more useful information of the query class. Even though the number of positive samples is lower at the first iteration, the fostering of the least used topics made by LTR 


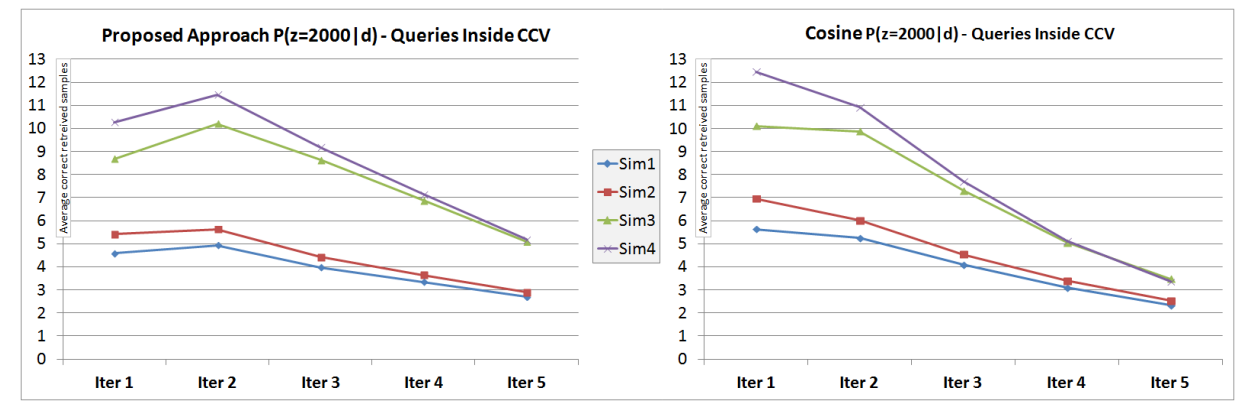

(a) Simulations when queries are from inside the CCV database.

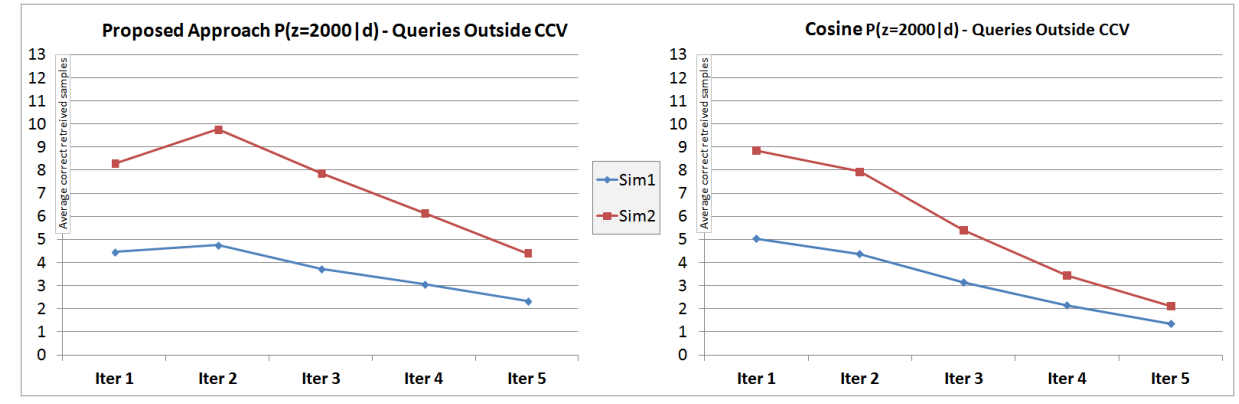

(b) Simulations when queries are from outside the CCV database.

Figure 8: Proposed approach (LTR) vs Cosine Similarity (CS). Average of correctly retrieved samples per iteration for the performed video retrieval simulations using the CCV database.

generates a user's feedback more meaningful because it includes samples with a broader variety of topics related to the query. Eventually, this variety of hidden patterns allows users to describe better the concept of interest though the feedback they provide.

\subsection{Computational complexity issues}

Regarding the computational burden, the results show a high performance of the LTR function with respect to the best tested methods in both $P(W \mid D)$ and $P(Z \mid D)$ spaces. LTR can process documents faster than the methods 
tested in the original BoW space $P(W \mid D)$ because the proposed function performs the ranking in the topic-model space $P(Z \mid D)$ and this space has usually a lower dimensionality than the former. For instance, in the CCV simulations the original feature space with 5000 words is reduced by LDA to a topic space with 2000 topics what means a $60 \%$ dimensionality reduction. Comparing LTR with the methods tested in $P(Z \mid D)$, the proposed function is able to obtain a good computational time as well. Despite the fact that EC-pzd is more efficient than LTR, the precision of the Euclidean distance in the latent space is so poor that the single competitor of LTR is CS-pzd.

According to the results, LTR tends to outperform the computational time obtained by CS-pzd. The proposed LTR function (Eq. (16)) summarises the whole query set in a single document (query summary), then a single scalar product is performed for each sample to be ranked. That is, the cost of obtaining the score of a document is $O\left(\left|D^{\prime}\right| K+K\right)=O\left(\left|D^{\prime}\right| K\right)$ where $\left|D^{\prime}\right|$ represents the size of the query set at a specific time moment and $K$ the number of topics. Note that topic weights $\left(\sum_{d_{i}} p\left(z \mid d_{i}\right)\right)$ in Eq. (16) are computed off-line. The CS function (Eq. (15)) uses the average cosine value for all the documents of the query, therefore it needs to compute $\left|D^{\prime}\right|$ scalar products, two magnitudes and a query cardinality per document to rank. That makes a total cost of $O\left(3\left|D^{\prime}\right| K+\left|D^{\prime}\right|\right)=O\left(\left|D^{\prime}\right| K\right)$. The asymptotic cost of both functions is the same but in practice LTR is able to achieve a better computational time because of the multiplicative constants.

The average computational time per query shown in the results corresponds to the cost of the ranking function itself, that is, the stage (iii) of Fig. 4. However, the RF scheme contains two more procedures that we should 
Table 5: Computational time of LDA and EMS for the CCV database.

\begin{tabular}{|c|c|c|c|c|c|c|c|}
\hline \multicolumn{4}{|c|}{ LDA (default parameters) } & \multicolumn{5}{c|}{ EMS (default parameters) } \\
\hline & $\mathbf{K}$ & Time & RAM & CCV - tst & AVG Time per Doc & EM Iters & MAX Iters \\
\cline { 2 - 8 }$y$ & 100 & 2 days & $0.40 \mathrm{~GB}$ & $P\left(z=100 \mid d^{\prime}\right)$ & $0.63 \mathrm{sec}$ & 475.55 & $2.23 \%$ \\
\cline { 2 - 8 } & 500 & 8 days & $1.20 \mathrm{~GB}$ & $P\left(z=500 \mid d^{\prime}\right)$ & $3.68 \mathrm{sec}$ & 548.83 & $4.34 \%$ \\
\cline { 2 - 8 } CCV (tra + tst) & 1000 & 15.5 days & $2.20 \mathrm{~GB}$ & $P\left(z=1000 \mid d^{\prime}\right)$ & $9.05 \mathrm{sec}$ & 662.76 & $5.49 \%$ \\
\cline { 2 - 8 } & 1500 & 23 days & $3.20 \mathrm{~GB}$ & $P\left(z=1500 \mid d^{\prime}\right)$ & $15.24 \mathrm{sec}$ & 668.72 & $6.26 \%$ \\
\cline { 2 - 8 } & 2000 & 30.5 days & $4.20 \mathrm{~GB}$ & $P\left(z=2000 \mid d^{\prime}\right)$ & $22.31 \mathrm{sec}$ & 711.14 & $6.94 \%$ \\
\hline
\end{tabular}

take into account: LDA in stage (i) and EMS in (ii). Table 5 presents the computational time of both procedures for the CCV database. In the case of LDA, we use a parallel version running in 24 Intel Xeon E5-2640 processors and in the case of EMS a single processor Intel Xeon E5-2640. As we can see, the topic extraction task is a very time-consuming process. Although LDA runs off-line, its cost may limit its usage in much larger databases. However, the proposed LTR function is independent of the topic extraction algorithm, therefore further improved methods could be used instead of LDA.

Related to the EMS function, the more topics the more costly the process. In the case of $\mathrm{CCV}$, the average time to represent an external query document in 2000 topics is over 20 seconds what seems noticeable higher compared with the costs of the ranking functions. However, this cost has to be taken as a preprocessing step although it is part of the ON-LINE - Query Definition stage. In the case of external queries, a pre-processing step is always required to represent those external samples in the same way the database was encoded in visual Bag of Words. Finally, note that these computational disadvantages of LDA and EMS are not exclusive for the proposed LTR function but for all the retrieval methods running in latent topic spaces.

In addition to computational time, Table 5 shows the convergence average 
values of the EMS function for the CCV database. As we can see the average number of EM iterations per document is below the considered default limit of 1000 and besides there is a small percentage of documents which actually reach this limit in the convergence process.

\subsection{Limitations of the proposed approach}

Although the presented LTR function has shown to outperform the rest of the tested methods, there are two points which have to be taken into account: (i) the topic extraction cost and (ii) the patterns diversity provided by LTR. Related to the first point, current topic extraction algorithms are still very costly and more research in that field is required to enable processing video collections with millions of videos. This is is not a limitation specifically of LTR but it is a drawback of all the ranking functions working in the latent topic space. However, the proposed approach has been designed isolating the off-line topic extraction process from the on-line retrieval task. This makes that further improvements on the topic extraction methods can be directly used by replacing LDA to extract the topics. The second point to be considered is the patterns diversity provided by LTR. The proposed retrieval approach has been designed assuming a wide semantic gap to deal with by means of a RF scheme, that is the typical situation in CBVR. As we have shown, the topic diversity provided by LTR at the top-ranking is able to provide a competitive advantage because it may obtain a more informative

feedback. However, this diversity is only useful when there is feedback itself because the user discards those samples related to useless patterns. That limits the effectiveness of LTR in those situations where there is not feedback at all. As we can see in Fig. 8, the precision gains of LTR over CS are 
obtained after the first user's feedback. That is, CS obtains a better precision than LTR at the first ranking iteration where there is not feedback just the query initialisation.

\section{Conclusions}

In this work, we have presented a novel interactive retrieval approach addressing the retrieval problem as a class discovery problem using latent topics. The sSpLSA model has been introduced to deduce the LTR probabilistic ranking function and the EMS procedure has been defined to enable external queries. Later, we have defined the proposed retrieval framework based on short-term relevance feedback. Finally, several retrieval simulations have been performed using three different datasets (PAL, CCV and TRECVID) and several of the most relevant retrieval methods in the literature.

One of the main conclusions that arises from the work is the importance of topic models to deal with the semantic gap in CBVR. Although topic models have shown to be helpful in many areas, they have not been traditionally considered useful in CBVR because of the special nature of the latent space. However, this work shows that (i) the hidden patterns defined by topics can be effectively used in video retrieval tasks and (ii) the proposed LTR ranking function is able to outperform the rest of the tested functions mainly because it has the same probabilistic nature than topic models.

The results of the work provide evidences about the viability of the proposed approach in terms of effectiveness and efficiency to deal with the semantic gap challenge in the CBVR field. In this domain, two users could provide the same query initialisation but referring to two different query 
concepts because each one is focusing on a different aspect. As a result, the feedback quality is an essential issue to find out about the query concept. As we have shown, the proposed LTR function promotes the least used topics and then it enriches the top-ranking with a more variety of related hidden patterns what eventually produces a more meaningful feedback.

Although results are encouraging, much more progress is needed to really address the semantic gap problem which involves several fields, from low level descriptors to high level understanding and user interaction. Specifically, further work is directed to extend the work in the following directions:

- Automatic strategies to set the most appropriate number of topics.

- Extension of the retrieval model to a long-term RF approach.

- Reduction of the computational time of the topic extraction task by applying quantisation methods in the initial object (video) space.

\section{References}

[1] S. Antani, A survey on the use of pattern recognition methods for abstraction, indexing and retrieval of images and video, Pattern Recognition 35 (2002) 945-965.

[2] A. F. Smeaton, Techniques used and open challenges to the analysis, indexing and retrieval of digital video, Information Systems 32 (2006) $545-559$.

[3] Y. Liu, D. Zhang, G. Lu, W.-Y. Ma, A survey of content-based image retrieval with high-level semantics, Pattern Recognition 40 (2007) 262282. 
[4] D. Zhang, M. M. Islam, G. Lu, A review on automatic image annotation techniques, Pattern Recognition 45 (2012) 346-362.

[5] G. Chechik, V. Sharma, U. Shalit, S. Bengio, Large scale online learning of image similarity through ranking, Journal of Machine Learning Research 11 (2010) 1109-1135.

[6] G.-H. Liu, Z.-Y. Li, L. Zhang, Y. Xu, Image retrieval based on microstructure descriptor, Pattern Recognition 44 (2011) 2123-2133.

[7] M. Arevalillo-Herrez, F. J. Ferri, J. Domingo, A naive relevance feedback model for content-based image retrieval using multiple similarity measures, Pattern Recognition 43 (2010) 619-629.

[8] R. d. S. Torres, A. X. Falcão, M. A. Gonçalves, J. a. P. Papa, B. Zhang, W. Fan, E. A. Fox, A genetic programming framework for content-based image retrieval, Pattern Recognition 42 (2009) 283-292.

[9] W. Ren, S. Singh, M. Singh, Y. S. Zhu, State-of-the-art on spatiotemporal information-based video retrieval, Pattern Recognition 42 (2009) 267-282.

[10] S. Tong, E. Chang, Support vector machine active learning for image retrieval, in: ACM International Conference on Multimedia, pp. 107118.

[11] K. Tieu, P. Viola, Boosting image retrieval, International Journal of Computer Vision 56 (2004) 17-36. 
[12] Y. Jiang, S. Bhattacharya, S. Chang, M. Shah, High-level event recognition in unconstrained videos, International Journal of Multimedia Information Retrieval 2 (2013) 73-101.

[13] D. Zhou, J. Weston, A. Gretton, O. Bousquet, B. Schölkopf, Ranking on data manifolds, in: Advances in Neural Information Processing Systems.

[14] Y. Yang, F. Nie, D. Xu, J. Luo, Y. Zhuang, Y. Pan, A multimedia retrieval framework based on semi-supervised ranking and relevance feedback, IEEE Transactions on Pattern Analysis and Machine Intelligence 34 (2012) 723-742.

[15] R. Zhang, Z. Zhang, Effective image retrieval based on hidden concept discovery in image database, IEEE Transactions on Image Processing 16 (2007) 562-572.

[16] D. G. Lowe, Distinctive image features from scale-invariant keypoints, International Journal of Computer Vision 60 (2004) 91-110.

[17] I. Laptev, On space-time interest points, International Journal of Computer Vision 64 (2005) 107-123.

[18] C. V. Cotton, D. P. W. Ellis, Audio fingerprinting to identify multiple videos of an event, in: IEEE International Conference on Acoustics, Speech and Signal Processing, pp. 2386-2389.

[19] J. Sivic, A. Zisserman, Video Google: A text retrieval approach to object matching in videos, in: International Conference on Computer Vision, volume 2, pp. 1470-1477. 
[20] H. Wang, C. Schmid, Action recognition with improved trajectories, in: IEEE International Conference on Computer Vision, pp. 3551-3558.

[21] C. Snoek, M. Worring, J. Gemert, J. Geusebroek, A. Smeulders, The challenge problem for automated detection of 101 semantic concepts in multimedia, ACM International Conference on Multimedia (2006).

[22] A. W. M. Smeulders, M. Worring, S. Santini, A. Gupta, R. Jain, Content-based image retrieval at the end of the early years, IEEE Transactions on Pattern Analysis and Machine Intelligence 22 (2000) 1349-1380.

[23] R. Fernandez-Beltran, F. Pla, An interactive video retrieval approach based on latent topics, in: International Conference on Image Analysis and Processing, pp. 290-299.

[24] D. M. Blei, Probabilistic topic models, Communications of the ACM 55 (2012) 77-84.

[25] A. Atreya, C. Elkan, Latent semantic indexing (lsi) fails for trec collections, SIGKDD Explor. Newsl. 12 (2011) 5-10.

[26] M. Minear, D. Park, A lifespan database of adult facial stimuli, Behavior Research Methods, Instruments, and Computers 36 (2004) 630-633.

[27] Y. G. Jiang, G. Ye, S. F. Chang, D. Ellis, A. C. Loui, Consumer video understanding: a benchmark database and an evaluation of human and machine performance, in: ACM International Conference on Multimedia Retrieval. 
[28] S. Ayache, G. Qunot, Trecvid 2007 collaborative annotation using active learning, in: Proceedings of the TRECVID 2007 Workshop.

[29] T. Hofmann, Unsupervised learning by probabilistic latent semantic analysis, Machine Learning 42 (2001) 177-196.

[30] D. Blei, A. Ng, M. Jordan, Latent dirichlet allocation, Journal of Machine Learning Research 3 (2003) 993-1022.

[31] J. Chang, S. Gerrish, C. Wang, J. L. Boyd-graber, D. M. Blei, Reading tea leaves: How humans interpret topic models, in: Advances in Neural Information Processing Systems 22, 2009, pp. 288-296.

[32] Y. Lu, Q. Mei, C. Zhai, Investigating task performance of probabilistic topic models: An empirical study of plsa and lda, Information Retrieval 14 (2011) 178-203.

[33] F.-F. Li, P. Perona, A bayesian hierarchical model for learning natural scene categories, in: IEEE Computer Society Conference on Computer Vision and Pattern Recognition, pp. 524-531.

[34] T. Joachims, Optimizing search engines using clickthrough data, in: Proceedings of the Eighth ACM SIGKDD International Conference on Knowledge Discovery and Data Mining, ACM, 2002, pp. 133-142.

[35] M. M. Deza, E. Deza, Encyclopedia of Distances, Springer Berlin Heidelberg, 2009.

[36] K. E. A. van de Sande, T. Gevers, C. G. M. Snoek, Evaluating color 
descriptors for object and scene recognition, IEEE Transactions on Pattern Analysis and Machine Intelligence 32 (2010) 1582-1596. 